\title{
A constitutive model for self-reinforced ductile polymer composites
}

\author{
S. Kazemahvazi ${ }^{\dagger 1}$, C. Schneider ${ }^{\dagger}$ and V.S. Deshpande ${ }^{\dagger}$ \\ $\dagger$ Department of Engineering, University of Cambridge, Cambridge, CB2 1PZ, UK. \\ $\$$ KTH, Department of Aeronautical and Vehicle Engineering, Stockholm, Sweden
}

\begin{abstract}
Self-reinforced polymer composites are gaining increasing interest due to their higher ductility compared to traditional glass and carbon fibre composites. Here we consider a class of PET composites comprising woven PET fibres in a PET matrix. While there is a significant literature on the development of these materials and their mechanical properties, little progress has been reported on constitutive models for these composites. Here we report the development of an anisotropic visco-plastic constitutive model for PET composites that captures the measured anisotropy, tension/compression asymmetry and ductility. This model is implemented in a commercial finite element package and shown to capture the measured response of PET composite plates and beams in different orientations to a high degree of accuracy.
\end{abstract}

Keywords: A. Thermoplastic resin, B. Anisotropy, C. Analytical modelling, C. Finite element analysis (FEA), Self-reinforced composite

${ }^{1}$ Corresponding author, Tel.: +44 (0) 7880989372

Email address: sk525@cam.ac.uk 


\section{Introduction}

A drawback with traditional fibre reinforced plastics (FRP) such carbon fibre reinforced plastics (CFRP) and glass fibre reinforced plastics (GFRP) is their low tensile failure strain for loading in fibre direction. Under tensile loading, the failure of these composites is usually catastrophic with little damage prior to ultimate failure. As a consequence, designs with these materials have high safety margins and also require costly structural health monitoring systems to be employed when such composites are used in safety critical applications.

There has been considerable recent interest in designing FRPs with higher ductilities. One approach is the use of a relatively new class of composites called single polymer composites (or self-reinforced composite) made from polymers such as polyethylene terephthalate (PET) and polypropylene (PP) [1-3]. These composites have been shown to have significantly higher ductilities compared to traditional CFRP and GFRP composites. As an example, the tensile failure strain of self-reinforced PET composites is $>10 \%$ which is an order of magnitude higher than the failure strain of e.g. GFRP (1.4\%) [4]. Most of the work reported to-date in these self-reinforced composites has focused on development of materials/manufacturing methods $[5,6]$ and the characterization of the mechanical properties for various single polymer materials [7-9]. Another important aspect with these group of materials is that the final mechanical properties of the material is highly dependent on manufacturing parameters such as consolidation temperature and pressure. The effect of manufacturing parameters on the 
mechanical properties of single polymer PP has e.g. been investigated by Alcock et al. $[10,11]$ and Hine et al. [12].

There has only been limited research on the modelling of the mechanical properties and behaviour of single polymer composites. Previous work has mainly focused on modelling the homogenized stiffness properties of the material by using a rule-ofmixture approach $[13,14]$. However, the application of single polymer composites in semi-structural and structural settings (e.g. such as the lattice sandwich cores developed by Schneider et al. [15]) requires the availability of design tools that includes more complete material constitutive models for performing structural calculations using finite element codes. Constitutive models such as the Hashin model [16], the Matzenmiller model [17] and the LaRC model [18] developed for CFRP and GFRP are unsuitable for the ductile self-reinforced composites because they: (i) are designed for elastic-brittle materials; (ii) cannot account for a rate sensitive plastic response and (iii) typically only model the in-plane response of composites.

In this study we will develop an anisotropic visco-plastic material model to capture the complex behaviour of ductile self-reinforced composites as described above. The outline of the paper is as follows. First we describe the PET composite material investigated in this study and report measurements of material properties in tension, compression and shear in different directions to fully characterise the orthotropic properties of these composites. Next, we describe an anisotropic visco-plastic model that is capable of capturing the measured anisotropic properties including tension/compression asymmetry and material rate dependence. The model is based on the homogenized properties of the composite material post consolidation and does 
therefore not require information on the material constituents (e.g. fibres/tape and matrix) properties prior to the consolidation process (these properties can change depending on processing conditions as discussed previously). Finally, structural tests on beams and plates of the composite in different orientations are reported. These measurements are compared with finite element predictions using the proposed constitutive model to demonstrate the fidelity of the constitutive model in capturing the complex structural behaviour of PET composites.

\section{Materials and manufacture}

The PET composites are made from commingled yarns comprising of 50\% high tenacity PET fibre (HTPET) with a melting temperature of $260^{\circ} \mathrm{C}$ and $50 \%$ PET fibres (LPET) with a lower melting temperature of $170^{\circ} \mathrm{C}$ that will subsequently be melted to form the matrix in the composite. These comingled yarns are then woven into a fabric with $80 \%$ of the yarns in the $x_{1}$ direction and only $20 \%$ in the $x_{2}$ direction as sketched in Fig.1. This woven fabric was supplied by Comfil®APS ${ }^{1}$ and is labelled by the supplier as unidirectional since the majority of the fibres lies in the $x_{1}$ direction. This fabric is then layered, with all layers stacked in the same direction, and consolidated into panels of the desired thickness in the $x_{3}$ direction under a pressure of 1.5 bar for 20 $\min$ at $220^{\circ} \mathrm{C}$ (i.e. a temperature that melts the LPET fibres to form the matrix but does not affect the HTPET fibres). Schneider et al. [4] gives a more detailed description of the manufacturing process and the individual properties of the HTPET fibres ( $E=15.2$ $\mathrm{GPa}$ ) and LPET matrix ( $E=3.0 \mathrm{GPa})$. For the sake of brevity these pressed composite panels shall be referred to as PET composites.

\footnotetext{
${ }^{1}$ Karolinelundsvej 2 - DK- 8883 Gjern, Denmark
} 


\section{Characterisation of mechanical properties}

The PET composites are highly anisotropic and here we report measurements to characterise the anisotropic elastic and inelastic properties. Three types of measurements are performed: (i) uniaxial tension in the $x_{1}$ and $x_{2}$ directions; (ii) uniaxial compression in the $x_{1}, x_{2}$ and $x_{3}$ directions and (iii) three-point bending of short and thick beams to measure shear responses in the $x_{1}-x_{2}, x_{1}-x_{3}$ and $x_{2}-x_{3}$ planes. For the shear tests, only a single repeat was performed to measure the qualitative response (the stiffness measurement is however confirmed to be the same in all 3 shear tests) while in all other tests at least 3 repeat tests were conducted to confirm the reproducibility of the results.

\subsection{Measurement protocol}

Tensile tests: Tensile tests were performed in the in-plane $x_{1}$ and $x_{2}$ directions using the dog bone shaped specimen sketched in Fig. 2a. The PET composites are highly anisotropic with a high tensile strength in the fibre directions but a relatively low shear strength. Hence the use of a test standard (such as that defined by ASTM D3039) results in failure by fibre pull-out at the grips and hence the specimen sketched in Fig. 2a developed by Russell et al [19] for the highly anisotropic polyethylene fibre composites was employed here. The applied tensile stress was defined using the load measured from the load cell of the test machine while the tensile strains were measured via a clip gauge on a central $12.5 \mathrm{~mm}$ gauge section of the specimen. In addition, in the initial elastic regime, the strains on the surface of the gauge section were recorded using the 
commercial digital image correlation (DIC) package GOM Aramis² and used to calculate the Poisson's ratio $v_{21}, v_{13}$ and $v_{23}$. All tests were conducted at an applied strain rate of $10^{-4} \mathrm{~s}^{-1}$ and unloading-reloading was also performed in order to measure the elastic moduli. No tensile tests were performed in the $x_{3}$-direction (thickness direction) as PET composites of sufficient thickness could not be manufactured in order to make tensile specimens of the appropriate shapes.

Compression tests: Two types of compression tests were conducted: (a) quasi-static tests at an applied strain rate of $10^{-4} \mathrm{~s}^{-1}$ and (b) high rate compression tests using a direct impact Kolsky bar at applied strain rates in the range $100 \mathrm{~s}^{-1} \leq \dot{\varepsilon} \leq 2000 \mathrm{~s}^{-1}$. All tests were conducted on cubes of specimens of side $H=13 \mathrm{~mm}$ (Fig. 2b). The quasi-static tests were conducted by compressing the cubes between lubricated rigid platens of a screw driven test machine in the $x_{1}, x_{2}$ and $x_{3}$ directions. Load measured from the machine load cell was used to define the applied stress and a laser gauge used to measure the platen displacement from which the applied strain is inferred. Unloading-reloading was also performed in order to measure the elastic moduli. The high strain rate Kolsky bar measurements were performed by compression tests in $x_{1}$ direction. Details of the Kolsky bar technique are given in [20]: briefly the compressive nominal stress is determined from strain measurements on the transmitter Kolsky bar and nominal strain for the imposed strain rate $v_{0} / H$ defined as $v_{0} t / H$ where $v_{0}$ is the velocity of the projectile that impact the specimens and time $t=0$ corresponds to the

\footnotetext{
${ }^{2}$ GOM Gmbh. User Manual: Aramis v6.3.0, Braunschweig, Germany, http://www.gom.com
} 
instant the projectile impacts the specimen. High speed photography was used to confirm the accuracy of the above definitions of strain rate and strain.

Shear tests: Three-point bend tests on short beams were conducted in order to measure the shear responses $\sigma_{12}-\gamma_{12}, \sigma_{13}-\gamma_{13}$ and $\sigma_{23}-\gamma_{23}$ roughly in accordance with the ASTM D2344 standard. Beams of dimension $13 \mathrm{~mm} \times 13 \mathrm{~mm} \times 78 \mathrm{~mm}$ were supported between rollers of diameter $3 \mathrm{~mm}$ spaced $50 \mathrm{~mm}$ apart and loaded at mid span by a $6 \mathrm{~mm}$ diameter roller (Fig. 2c) in a screw-driven test machine. The specimens were cut such that the longitudinal and transverse beam directions $X_{1}$ and $X_{2}$, respectively (Fig. 2c) were aligned with the material directions $x_{i}$ and $x_{j}$ to measure the $\sigma_{i j}-\gamma_{i j}$ response. The applied load $P$ on the central roller was measured from the load cell of the test machine and the corresponding applied displacement $\delta$ to the roller measured via a laser interferometer. The applied shear stress is defined as $3 P /(4 A)$ (based on the elastic value of the maximum shear stress) where $A=13 \times 13 \mathrm{~mm}^{2}$, is the beam cross-sectional area. The shear strain has been measured directly using the DIC system. All tests were conducted at an applied strain rate $\dot{\gamma} \equiv 2 \dot{\delta} / L=10^{-4} \mathrm{~s}^{-1}$ where $L=50 \mathrm{~mm}$ is the span of the beam.

\subsection{Measured material properties}

The measured uniaxial true tensile stress ${ }^{3}$ versus logarithmic strain curves $\left(\sigma_{11}-\varepsilon_{11}\right.$ and $\sigma_{22}-\varepsilon_{22}$ ), at an applied strain rate of $10^{-4} \mathrm{~s}^{-1}$ are plotted in Fig. 3a. In both cases

\footnotetext{
${ }^{3}$ True stress is calculated from the nominal stress assuming incompressible deformation. While deformation is not incompressible in the elastic regime, the error introduced by this approximation is negligible in that regime as the elastic strains are small. The incompressible assumption is accurate in the plastic regime.
} 
after an initial elastic response the tensile response of the PET composites is strain hardening with tensile failure due to fibre fracture at $13 \%$ and $10 \%$ applied strains for loading in the $x_{1}$ and $x_{2}$ directions, respectively. This ductile plastic response is in contrast to the typical elastic-brittle tensile responses observed for CFRP and GRP composites for loading in the fibre directions. Both the elastic modulus and the yield strength of the PET composites is higher in the $x_{1}$ directions as a larger fraction of the fibres are aligned in this direction. The measured Young's moduli $\left(E_{11}\right.$ and $\left.E_{22}\right)$ from the unloaded tests (not included for the sake of clarity in Fig. 3a) are listed in Table 1. The Poisson's ratio measured from these tensile tests are plotted in Fig. $3 \mathrm{~b}$ as a function of the applied strains $\left(v_{12}-\varepsilon_{11}, v_{13}-\varepsilon_{11}\right.$ and $\left.v_{23}-\varepsilon_{22}\right)$. It is clear that the measured ratios are approximately constant in the elastic range of the loading. These measured values are also included in Table 1.

The measured uniaxial compressive true stress versus logarithmic strain responses $\sigma_{11}-\varepsilon_{11}, \sigma_{22}-\varepsilon_{22}$ and,$\sigma_{33}-\varepsilon_{33}$ are included in Fig. 3a for loading at an applied strain rate of $10^{-4} \mathrm{~s}^{-1}$. In all cases, we observe an initial elastic response followed by a plateau stress for loading in the $x_{1}$ and $x_{2}$ directions. This plateau strength is approximately equal for compression in both the $x_{1}$ and $x_{2}$ directions as the compressive strength of the fibres (in the fibre direction) is approximately the same as the matrix compressive strength. At approximately $10 \%$ compressive strain the specimens start to soften significantly due to fibre buckling that leads to ply delamination. By contrast, compression in the $x_{3}$ direction results in a strain hardening response after the initial elastic regime. The compressive true stress rises from the initial yield value of approximately $100 \mathrm{MPa}$ to $200 \mathrm{MPa}$ at an applied compressive strain of approximately $25 \%$ at which point the specimen fails catastrophically by 
tensile fibre fracture. We expect that this tensile failure occurs via the generation of indirect tension in a manner similar to that observed for polyethylene fibre composites [21]. The indirect tension mechanism is summarized as follows. When the material is loaded the $x_{3}$ direction, the fibres that are oriented in the $x_{1}$ direction (80\% of the fibres) want to expand transversally in the $x_{2}$ direction. The expansion is however restricted by the fibres that are oriented in the $x_{2}$ direction ( $20 \%$ of the fibres) via shear lag which results in the $x_{2}$ direction fibres being loaded in tension indirectly. As the compressive load in the $x_{3}$ direction increases the tension loads in the $x_{2}$ direction will thus increase which eventually leads to fibre rupture.

The measured true compressive stress versus logarithmic strain curves at the high applied nominal strain rates in the range $100 \mathrm{~s}^{-1} \leq \dot{\varepsilon} \leq 2000 \mathrm{~s}^{-1}$ are plotted in Fig. $4 \mathrm{a}$ for loading in the $x_{1}$ direction (for the sake of clarity the negative of the stress and strain are plotted). Here we define the logarithmic strain as $\log \left(1+v_{0} t / H\right)$ and the true stress again calculated from the measured load assuming incompressible deformation. Unlike under quasi-static conditions, no distinct plateau in the stress versus strain response is observed. We attribute this to the early delamination of the specimens: montages of images showing the deformation of the specimen compressed at $\dot{\varepsilon}=$ $2000 \mathrm{~s}^{-1}$ and $10^{-4} \mathrm{~s}^{-1}$ are included in Fig. 4a and show that early delamination sets in the specimen deformation at the high strain rate. The measured peak strengths $\sigma_{p}$ (normalised by the quasi-static strength $\sigma_{0}^{Y}=104 \mathrm{MPa}$ in the $x_{1}$ direction) for compression in the $x_{1}$ direction at strain rates ranging from the quasi-static value of $10^{-4} \mathrm{~s}^{-1}$ to $2000 \mathrm{~s}^{-1}$ are plotted in Fig. $4 \mathrm{~b}$. It is clear that the strain rate sensitivity of the response of the PET composites is low with the peak strength rising by only $70 \%$ when the applied strain rate is varied by 7 orders of magnitude. 
The shear stress versus engineering shear strain $\left(\sigma_{12}-\gamma_{12}, \sigma_{13}-\gamma_{13}\right.$ and $\left.\sigma_{23}-\gamma_{23}\right)$ responses of PET composites are plotted in Fig. 5. In all cases, the PET composites show a linear elastic response followed by progressive localised interlaminar shearing until catastrophic failure occurs. The interlaminar shear localisation results in a pseudoductile shear behaviour in all directions but in the $x_{1}-x_{3}$ direction. Contour plots of engineering shear strains on the surface of the specimen (measured using DIC) near the initial yield point are included as an inset in Fig. 5: the contour plot of $\gamma_{13}$ most clearly shows the intense shear localisation on the beam mid-plane just prior to failure. The measured shear moduli (using unloading measurements not included in Fig. 5 for the sake of clarity) are listed in Table 1.

\section{Constitutive model for ductile anisotropic PET composites}

The mechanical properties of the orthotropic PET composites described in Section 3.2 show that these composites are significantly more ductile compared to typical glass fibre or carbon fibre composites. Moreover, the strain hardening response of these ductile materials displays significant tension/compression asymmetry. Most constitutive models for composites such as the Hashin model [16] are designed for elastic-brittle composites and inherently unsuited to model the ductile PET composites. Here we propose a model for the PET composites that not only accounts for their anisotropy and ductility but is also capable of modelling the observed tension/compression asymmetry and the material anisotropy.

Consider the composite sketched in Fig. 1 with the woven fibers lying in the $x_{1}-x_{2}$ plane (the $x_{1}$ direction is taken to be the direction with the higher proportion of fibres as sketched in Fig. 1). The total strain rate $\dot{\varepsilon}_{i j}$ is decomposed into an elastic $\left(\dot{\varepsilon}_{i j}^{e}\right)$ and plastic $\left(\dot{\varepsilon}_{i j}^{p}\right)$ part such that 
$\dot{\varepsilon}_{i j}=\dot{\varepsilon}_{i j}^{e}+\dot{\varepsilon}_{i j}^{p}$

where the overdot indicates differentiation with respect to time. The micro-structure dictates that the composite is elastically orthotropic and hence the elastic strain is related to the Cauchy stress via

$$
\left(\begin{array}{c}
\varepsilon_{11}^{e} \\
\varepsilon_{22}^{e} \\
\varepsilon_{33}^{e} \\
\varepsilon_{23}^{e} \\
\varepsilon_{31}^{e} \\
\varepsilon_{12}^{e}
\end{array}\right)=\left(\begin{array}{cccccc}
\frac{1}{E_{11}} & -\frac{v_{21}}{E_{22}} & -\frac{v_{31}}{E_{33}} & 0 & 0 & 0 \\
-\frac{v_{12}}{E_{11}} & \frac{1}{E_{22}} & -\frac{v_{32}}{E_{33}} & 0 & 0 & 0 \\
-\frac{v_{13}}{E_{11}} & -\frac{v_{23}}{E_{22}} & \frac{1}{E_{33}} & 0 & 0 & 0 \\
0 & 0 & 0 & \frac{1}{2 G_{23}} & 0 & 0 \\
0 & 0 & 0 & 0 & \frac{1}{2 G_{31}} & 0 \\
0 & 0 & 0 & 0 & 0 & \frac{1}{2 G_{12}}
\end{array}\right)\left(\begin{array}{c}
\sigma_{11} \\
\sigma_{33} \\
\sigma_{23} \\
\sigma_{31} \\
\sigma_{12}
\end{array}\right),
$$

where $E_{i j}, G_{i j}$ and $v_{i j}$ are the Young's moduli, shear moduli and Poisson's ratios in the different direction (the subscripts $i j$ here refer to the directions and $E_{i j}, G_{i j}$ and $v_{i j}$ are not tensor quantities). Orthotropic symmetry considerations dictate that there are only 9 independent elastic constants with $v_{21} / E_{22}=v_{12} / E_{11}, v_{31} / E_{33}=v_{13} / E_{11}$ and $v_{32} / E_{33}=v_{23} / E_{22}$

Motivated by the rate sensitivity of the PET composites (Fig. 4b) we propose a viscoplastic model for the plastic component of the deformation. For the sake of simplicity (and also to ensure positive plastic dissipation) we shall assume associated plastic flow and hence begin by defining a viscoplastic potential $\Phi$ in terms of an effective stress $\hat{\sigma}$ as

$\Phi=\frac{\varepsilon_{0} \sigma_{0}}{m+1}\left(\frac{\widehat{\sigma}}{\sigma_{0}}\right)^{m+1}$ 
where $\dot{\varepsilon_{0}}, \sigma_{0}$ and $m$ are the reference strain rate, reference stress (shown subsequently to be equal to the quasi-static yield strength in the $x_{1}$ direction) and strain rate sensitivity exponent, respectively. The plastic strain rate is then given as

$\dot{\varepsilon}_{i j}^{p} \equiv \frac{\partial \Phi}{\partial \sigma_{i j}}=\dot{\varepsilon}_{0}\left(\frac{\widehat{\sigma}}{\sigma_{0}}\right)^{m} \frac{\partial \widehat{\sigma}}{\partial \sigma_{i j}}$.

It now remains to specify a functional form for $\hat{\sigma}$. The measurements reported in Section 3.2 suggest incompressible plastic deformation of the composites and hence we choose that $\hat{\sigma}$ is invariant to an applied pressure. Hill [22] suggested a form of an effective stress for a plastically orthotropic metal that is invariant to the applied pressure. Here we choose the Hill [22] effective stress formula to define $\hat{\sigma}$ as

$\widehat{\sigma}^{2}=A\left(\sigma_{11}-\sigma_{22}\right)^{2}+B\left(\sigma_{22}-\sigma_{33}\right)^{2}+C\left(\sigma_{33}-\sigma_{11}\right)^{2}+$

$D \sigma_{12}^{2}+E \sigma_{23}^{2}+F \sigma_{13}^{2}$

where the parameters $A-F$ define the current state of the plastic anisotropy. This completes the specification of the constitutive model. We proceed to discuss the calibration of this model.

\subsection{Calibration of model parameters}

The nine elastic coefficients $\left(E_{11}, E_{22}, E_{33}, v_{12}, v_{13}, v_{23}, G_{12}, G_{23}, G_{13}\right)$ are directly measured from the uniaxial compression/tension and shear tests in the different directions detailed in Section 3.2. These measured parameters are listed in Table 1. The relation of the plastic parameters $(A-F), \sigma_{0}, \dot{\varepsilon_{0}}$ and $m$ to the material tests described in Section 3.2 requires further detailing.

Consider first a uniaxial tension/compression test on the composite in the $x_{1}$-direction performed at an applied plastic strain rate of magnitude $\dot{\varepsilon}_{0}$. The measured magnitude of 
the applied uniaxial stress $\sigma_{11}$ versus plastic strain $\varepsilon_{11}^{p}$ response is denoted as $Y_{11}\left(\varepsilon_{11}^{p}\right)$. Equations (4)-(5) then specify that

$\dot{\varepsilon}_{11}^{p}=\dot{\varepsilon}_{0}\left(\frac{Y_{11}}{\sigma_{0}}\right)^{m}(A+C)^{\frac{m+1}{2}} \operatorname{sign}\left(\sigma_{11}\right)$.

We then define the reference stress $\sigma_{0}=Y_{11}\left(\varepsilon_{11}^{p}\right)$, which then implies that $A+C=1$. As a consequence we can now specify different strain hardening responses in tension and compression, i.e. in general $Y_{11}\left(\varepsilon_{11}^{p}\right) \neq Y_{11}\left(-\varepsilon_{11}^{p}\right)$ except of course at initial yield $\left(\varepsilon_{11}^{p}=0\right), \sigma_{0}$ takes a unique value. Now consider a uniaxial compression test in the $x_{1^{-}}$ direction at some arbitrary value of the applied strain rate. The plastic strain rate $\dot{\varepsilon}_{11}^{p}$ is related to the applied stress $\sigma_{11}$ via

$\dot{\varepsilon}_{11}^{p}=\dot{\varepsilon}_{0}\left(\frac{\left|\sigma_{11}\right|}{\sigma_{0}}\right)^{m} \operatorname{sign}\left(\sigma_{11}\right)$

and thus it is clearly seen that $m$ is the power-law exponent relating the applied stress to the plastic strain rate. The measured rate sensitivity of the PET composites is plotted in Fig. $4 \mathrm{~b}$ and we include in Fig. $4 \mathrm{~b}$ the curve $\varepsilon^{\cdot} / \dot{\varepsilon}_{0}=\left(\sigma_{p} / \sigma_{0}^{Y}\right)^{m}$ with $\dot{\varepsilon}_{0}=10^{-4} \mathrm{~s}^{-1}$ and $\sigma_{0}^{Y}=104 \mathrm{MPa}$ a strain rate exponent $m \approx 32$, fits the measurements with good accuracy.

It now remains to provide calibration formulae for the coefficients $A$ through $F$. Analogous to $Y_{11}\left(\varepsilon_{11}^{p}\right)$, define $Y_{22}\left(\varepsilon_{22}^{p}\right)$ and $Y_{33}\left(\varepsilon_{33}^{p}\right)$ as the uniaxial tension/compression applied stress (magnitude) versus plastic curves in the $x_{2}$ and $x_{3}$ directions, respectively at an applied plastic strain rate of $\dot{\varepsilon}_{0}$. Then using the plastic normality relation (4) we have analogous to Eq. (6)

$\dot{\varepsilon}_{22}^{p}=\dot{\varepsilon}_{0}\left(\frac{Y_{22}}{Y_{11}}\right)^{m}(A+B)^{\frac{m+1}{2}} \operatorname{sign}\left(\sigma_{22}\right)$,

and 


$$
\dot{\varepsilon}_{33}^{p}=\dot{\varepsilon}_{0}\left(\frac{Y_{33}}{Y_{11}}\right)^{m}(B+C)^{\frac{m+1}{2}} \operatorname{sign}\left(\sigma_{33}\right) .
$$

Recalling that $\left|\dot{\varepsilon}_{22}^{p}\right|=\left|\dot{\varepsilon}_{33}^{p}\right|=\dot{\varepsilon}_{0}$ and that $A+C=1$, we get upon simplification

$A=\frac{1}{2}\left[\left(\frac{Y_{11}}{Y_{22}}\right)^{\frac{2 m}{m+1}}-\left(\frac{Y_{11}}{Y_{33}}\right)^{\frac{2 m}{m+1}}+1\right]$

and

$B=\frac{1}{2}\left[\left(\frac{Y_{11}}{Y_{22}}\right)^{\frac{2 m}{m+1}}+\left(\frac{Y_{11}}{Y_{33}}\right)^{\frac{2 m}{m+1}}-1\right]$.

Similar arguments give the calibration of the coefficients $D$ through $F$ as

$D=\left(\frac{Y_{11}}{Y_{12}}\right)^{\frac{2 m}{m+1}}$

$E=\left(\frac{Y_{11}}{Y_{23}}\right)^{\frac{2 m}{m+1}}$, and

$F=\left(\frac{Y_{11}}{Y_{13}}\right)^{\frac{2 m}{m+1}}$

where $Y_{12}\left(\varepsilon_{12}^{p}\right), Y_{23}\left(\varepsilon_{23}^{p}\right)$ and $Y_{13}\left(\varepsilon_{13}^{p}\right)$ are the shear stress magnitude versus applied plastic shear strain curves performed at an applied plastic shear strain of magnitude $\dot{\varepsilon}_{0}$.

The material property measurements detailed in Section 3.2 provide all the information for the calibration of all the plastic material constants. The quasi-static tests were all performed at an applied strain rate of $10^{-4} \mathrm{~s}^{-1}$ and hence we take $\dot{\varepsilon}_{0}=10^{-4} \mathrm{~s}^{-1}$ and the curves $Y_{i j}\left(\varepsilon_{i j}^{p}\right)$ are inferred from the stress versus strain curves plotted in Figs. 3 and 5 as follows. In Figs. 3 and 5 the applied stress versus total strains curves are plotted: while $Y_{i j}=\left|\sigma_{i j}\right|$, the plastic strains are evaluated using the relations 
$\varepsilon_{i j}^{p}= \begin{cases}\varepsilon_{i j}-\frac{\sigma_{i j}}{E_{i j}} & \text { for } i=j \\ \varepsilon_{i j}-\frac{\sigma_{i j}}{2 G_{i j}} & \text { for } i \neq j .\end{cases}$

This completes the specification of the 3D constitutive model for PET composites and the method to calibrate the required coefficients.

\subsection{Implementation of constitutive law into a commercial finite element package}

In order to validate the constitutive law, it has been implemented as a user material (VUMAT) into the explicit version of the commercial finite element (FE) package ABAQUS. Comparisons between the measured and predicted stress versus strain curves for the various loading conditions discussed in Section 3.2 are shown in Figs. 3 and 5. Recall that the measurements are used to calibrate the model and hence the comparisons in Figs. 3 and 5 are essentially a check on the implementation of the model in the FE code. The predictions are in very good agreement with the experiments for all load cases $^{4}$. It is worth noting that the developed model only captures elastic-plastic deformation and not failure. This simplification works well in all principal loading directions (up to at-least $10 \%$ strains) accept for $\sigma_{13}$ loading in which case the composite is brittle with a low failure strain. The initiation of failure can be implemented into the model by e.g. assuming an uniaxial anisotropic maximum strain criterion (as will be shown in coming structural test) while the modelling of damage evolution will require

\footnotetext{
${ }^{4}$ The tensile response in the $x_{3}$ direction was not measured for the reasons explained in Section 3.1 and hence we assume here that there is no asymmetry between the tensile and compressive responses in the $x_{3}$ direction. For the sake of completeness, the predicted tensile $\sigma_{33}$ versus $\varepsilon_{33}$ response in shown in Fig. 3a as inferred from measured compressive response.
} 
considerable more experimental and modelling effort to be captured with sufficient fidelity.

\section{Structural tests on PET composites}

In order to investigate the fidelity of the constitutive model proposed above, we here report two types of structural loading tests: (i) three-point bending of simply supported beams with two different orientations of the PET composites and (ii) central loading of a clamped rectangular plate.

\subsection{Three-point bending of beams}

Beams of length $L=110 \mathrm{~mm}$, width $W=20 \mathrm{~mm}$ and thickness $t=6 \mathrm{~mm}$ were cut from the PET composite sheets in two orientations as shown in the inset in Fig. 6a.

Define a global co-ordinate system such that $X_{3}$ is in the thickness direction of the beam and $X_{1}$ along the length of the beam as sketched in Fig. 6a. In both orientations the $X_{3}$ and $x_{3}$ axes co-incided but the $x_{1}$ and $x_{2}$ material directions were oriented in the specimen as follows:

(i) Orientation I: The $x_{1}$ material direction was aligned with the $X_{1}$-axis.

(ii) Orientation II: The $x_{1}$ material direction was oriented at $40^{\circ}$ with respect to the $X_{1}$-axis.

The beams were supported on cylindrical rollers of diameter $20 \mathrm{~mm}$ spaced $90 \mathrm{~mm}$ and loaded centrally by a roller also of diameter $20 \mathrm{~mm}$ in a screw-driven test machine. The central roller was displaced at a rate $\dot{\delta}=0.017 \mathrm{~mm} \mathrm{~s}^{-1}$ and the applied load $P$ was measured via the load cell of the test machine and a laser interferometer used to determine the central roller displacement $\delta$.

The measured $P$ versus $\delta$ curves for the two orientations of the PET composite beams are plotted in Fig. 6b. In both cases the beams display an initial elastic response 
followed by a mildly hardening plastic regime. Both the modulus and bending strength of the beam are higher in orientation I: the $x_{1}$ material direction along which the majority of the fibres lie are aligned with the longitudinal axis of the beam in this orientation and this results in the higher stiffness and bending strength of the beams in orientation I.

A three-dimensional FE model of the beam was constructed in ABAQUS using cubes of 8-noded linear brick elements (C3D8R in ABAQUS notation) of size $0.3 \mathrm{~mm}$ (i.e. 20 elements through the thickness of the beam). Using symmetry only half of the beam was modelled with symmetry boundary conditions imposed at mid-span. The loading rollers were modelled as analytical rigid bodies and contact between the rollers and the beam was modelled using the ABAQUS general contact algorithm with a friction coefficient of 0.3. Loading was prescribed as in the experiments by displacing the central roller at a rate $\dot{\delta}=0.017 \mathrm{~mm} \mathrm{~s}^{-1}$ and the PET composite that the beam was made from was modelled via the material model and material constants described in Section 4. Predictions of the $P$ versus $\delta$ are included in Fig. 6b and show excellent agreement with the measurements for both beam orientations.

\subsection{Central loading of clamped plates}

Rectangular plates of thickness $5 \mathrm{~mm}$ and in-plane dimensions $170 \mathrm{~mm} \times 145 \mathrm{~mm}$ were cut from the PET composite plates such that the $x_{1}$ material direction was aligned with side of length $145 \mathrm{~mm}$. These plates were clamped in the aluminium frame sketched in Fig. 7a using sixteen M5 bolts such that the free-span of the plate was $120 \mathrm{~mm} \times 95 \mathrm{~mm}$ with the $x_{1}$ material direction aligned with the side of length 95 $\mathrm{mm}$. The plate was loaded at its geometrical center by a hemispherical indenter of diameter $50 \mathrm{~mm}$ displaced transverse to the plate (i.e. in the $x_{3}$ material direction) at an 
applied displacement rate $\dot{\delta}=0.017 \mathrm{~mm} \mathrm{~s}^{-1}$ in a screw driven test machine. The applied load $P$ was measured via the load cell of the test machine and the displacement $\delta$ of the indenter measured using a laser interferometer. The measured $P$ versus $\delta$ curve is included in Fig. $7 \mathrm{~b}$ and two knees in the curve are observed. At $P \approx 2 \mathrm{kN}$ there is a small decrease in the slope $d P / d \delta$ associated with the onset of plasticity in the PET composite plate while at $P \approx 12 \mathrm{kN}$ fibres in the $x_{2}$ material direction fracture under the indenter causing a sudden decrease in $d P / d \delta$. With increasing deflection $\delta$, the slope of the $P$ versus $\delta$ curve again increases as the plate starts to pull-in from the supports (i.e. the stresses consequently re-distribute).

A 3D FE model of the free-span of the plate (i.e. the plate of dimensions $120 \mathrm{~mm} \times$ $95 \mathrm{~mm}$ ) was constructed in ABAQUS using 8-noded linear brick elements (C3D8R). Only quarter of the plate was modelled using symmetry considerations with symmetry boundary conditions imposed along the two central mid-planes and clamped boundary conditions (i.e. all degrees of freedom constrained) along the two side edges. Thus, the portion of the plate within the supports and any pull-in from the supports was not modelled. The indenter was modelled as an analytical rigid body and frictionless contact between the indenter and plate was included via the ABAQUS general contact option. Again, the PET composite was modelled via the constitutive law and material parameters described in Section 4. The predicted $P$ versus $\delta$ curve is included in Fig. 7b. The initial stiffness (i.e. $d P / d \delta$ for $P<2 \mathrm{kN}$ ) is higher than that measured: we attribute this to the fact that the pull-in from the supports that occurs in the experiments is not included in the model. Subsequently, the FE model captures the response with good accuracy. However, recall that at $P \approx 12 \mathrm{kN}$ fibre fracture in the $x_{2}$ material direction occurs. This failure mode is not included in the FE model which causes the 
model to loose accuracy after this point. Notwithstanding, the FE model is adequate to predict the onset of failure. At an applied load of $P=12.3 \mathrm{kN}$ (i.e. the load at which fibre fracture was observed in the experiments), the FE model predicts a maximum tensile strain of $10.3 \%$ in the $x_{2}$ material direction. This value is consistent with the failure strain measured in the tensile tests in the $x_{2}$ material direction (Fig. 3a). Based on the structural measurements and simulations reported here we conclude that the constitutive model is capable of accurately predicting the response of PET composite structures under a variety of complex loading conditions up to the onset of fibre fracture. Moreover, it is also capable of accurately predicting the initiation of fibre fracture based on a tensile strain criterion.

\section{Concluding remarks}

The anisotropic material properties of ductile self-reinforced PET fibre composites have been measured and a constitutive model for such composites proposed. The constitutive model captures the elastic and plastic anisotropy of the composites in addition to the measured tension/compression asymmetry and material rate dependence. The constitutive model was implemented in a finite element code and used to make predictions of the structural response of PET composite plates and beams in different orientations. Comparisons of the measurements and predictions confirmed the fidelity of the constitutive model to capture the complex anisotropic response of the PET composites under large deformations.

While the model in its current state is adequate to predict the response of PET composite structures, it can also be readily complemented to include temperature dependent material properties. This will expand the applicability of the model to 
simulate hot forming manufacturing processes that are becoming increasingly common for thermoplastic self-reinforced composites.

\section{Acknowledgements}

We are grateful to the Defence Advanced Research Projects Agency (Grant Number W91CRB-10-1-005) for the financial support for this research. C. Schneider was funded by the Centre of ECO2 Vehicle Design and the Swedish Agency for Innovation Systems (VINNOVA). The authors would like to acknowledge the support from the material supplier Comfil.

\section{References}

[1] J. Karger-Kocsis, T. Bárány, Single-polymer composites (SPCs): Status and future trends, Composites Science and Technology, Volume 92, 2014, pp. 77-94

[2] C. Gao, L. Yu, H. Liu, L. Chen, Development of self-reinforced polymer composites, Progress in Polymer Science, Volume 37, 2012, pp. 767-780

[3] Á. Kmetty, T. Bárány, J. Karger-Kocsis, Self-reinforced polymeric materials: A review, Progress in Polymer Science, Volume 35, 2010, pp. 1288-1310

[4] C. Schneider, S. Kazemahvazi, M. Åkermo, D. Zenkert, Compression and tensile properties of self-reinforced poly(ethylene terephthalate)-composites, Polymer Testing, Volume 32, 2013, pp. 221-230

[5] J. Wang, J. Chen, P. Dai, Polyethylene naphthalate single-polymer-composites produced by the undercooling melt film stacking method, Composites Science and Technology, Volume 91, 2014, pp. 50-54

[6] J.M. Zhang, T. Peijs, Self-reinforced poly(ethylene terephthalate) composites by hot consolidation of Bi-component PET yarns, Composites Part A: Applied Science and Manufacturing, Volume 41, 2010, pp. 964-972 
[7] B. Alcock, N.O. Cabrera, N.-M. Barkoula, J. Loos, T. Peijs, The mechanical properties of unidirectional all-polypropylene composites, Composites Part A: Applied Science and Manufacturing, Volume 37, 2006, pp. 716-726

[8] Y. Swolfs, L. Crauwels, E.V. Breda, L. Gorbatikh, P. Hine, I. Ward, I. Verpoest, Tensile behaviour of intralayer hybrid composites of carbon fibre and selfreinforced polypropylene, Composites Part A: Applied Science and Manufacturing, Volume 59, 2014, pp. 78-84

[9] B. Alcock, N.O. Cabrera, N.-M. Barkoula, Z. Wang, T. Peijs, The effect of temperature and strain rate on the impact performance of recyclable allpolypropylene composites, Comp. Part B: Eng., Volume 39, 2008, pp. 537-547

[10] B. Alcock, N.O. Cabrera, N.-M. Barkoula, A.B. Spoelstra, J. Loos, T. Peijs, The mechanical properties of woven tape all-polypropylene composites, Composites: Part A, Volume 38, 2007, pp. 147-161.

[11] B. Alcock, N.O. Cabrera, N.M. Barkoula, T. Peijs, The effect of processing conditions on the mechanical properties and thermal stability of highly oriented PP tapes, European Polymer Journal, 45, 2009, pp. 2878-2894.

[12] P.J. Hine, I.M. Ward, N.D. Jordan, R. Olley, D.C. Bassett, The hot compaction behaviour of woven oriented polypropylene fibres and tapes. I. Mechanical properties, Polymer, Volume 44, 2003, pp. 1117-1131.

[13] Y. Swolfs, W. Van den fonteyne, J. Baets, I. Verpoest, Failure behaviour of selfreinforced polypropylene at and below room temperature, Comp. Part A, Volume 65, 2014, pp. 100-107.

[14] I.M. Ward, P.J. Hine, The science and technology of hot compaction, Polymer, Volume 45, 2004, 1413-1427. 
[15] C. Schneider, M.N. Velea, S. Kazemahvazi, D. Zenkert, Compression properties of novel thermoplastic carbon fibre and poly-ethylene terephthalate fibre composite lattice structures, Materials and Design, Volume 65, 2015, pp. 11101120.

[16] Z. Hashin, Failure criteria for unidirectional fiber composites, J Appl Mech, 47 (1980), pp. 329-334

[17] A. Matzenmiller, J. Lubliner, R.L. Taylor, A constitutive model for anisotropic damage in fiber-composites, Mechanics of Materials, 20, 1995, 125-152.

[18] P. Maimi', P.P. Camanho, J.A. Mayugo, C.G. Da'vila, A continuum damage model for composite laminates: Part I - Constitutive model, Mechanics of Materials, 39, 2007, 897-908.

[19] B.P. Russell, K. Karthikeyan, V.S. Deshpande, N.A. Fleck, The high strain rate response of Ultra High Molecular-weight Polyethylene: From fibre to laminate, International Journal of Impact Engineering, Volume 60, 2013, pp. 1-9

[20] S. Kazemahvazi, B.P. Russell, D. Zenkert, Impact of carbon fibre/epoxy corrugated cores, Composite Structures, Volume 94, 2012, pp. 3300-3308

[21] J.P. Attwood, S.N. Khaderi, K. Karthikeyan, N.A. Fleck, M.R. O'Masta, H.N.G. Wadley, V.S. Deshpande, The out-of-plane compressive response of image composites, J Mechanics Physics of Solids, Volume 70, 2014, pp. 200-226

[22] R. Hill. A theory of the yield and plastic flow of anisotropic metals. Proc. Roy. Soc. London. Ser. A, 193, 1948, pp. 281-297 


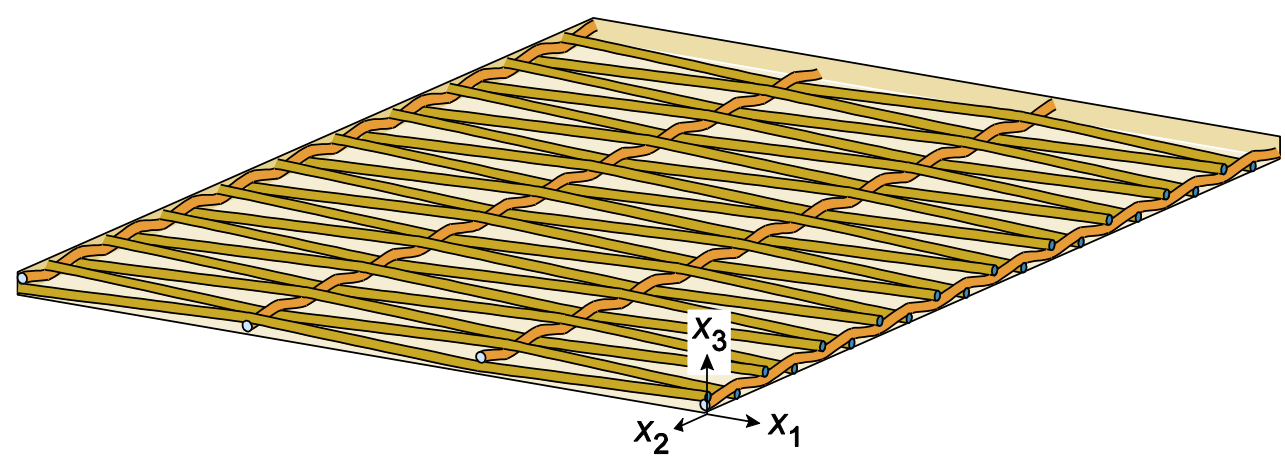

Figure 1: Sketch of the micro-structure of the fabric comprising comingled PET yarns. The definition of the material axes $x_{i}$ is included and the sketch shows that $80 \%$ of the PET yarns lie in the $x_{1}$ direction. 

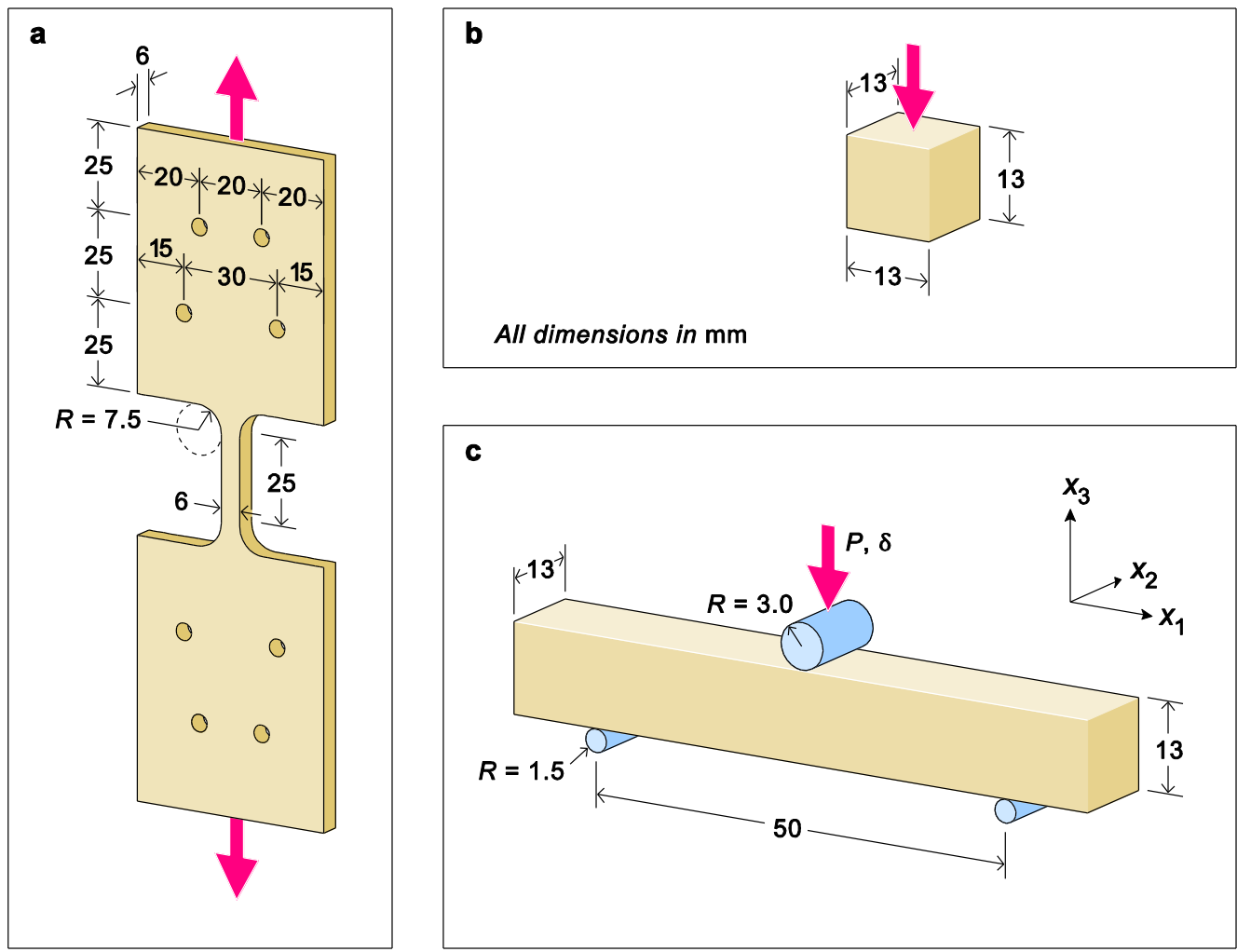

Figure 2: Sketches of the three types of specimens used to measure the anisotropic properties of the PET composites. The sketches are labelled with key parameters and all dimensions are in mm. (a) Tensile test specimen; (b) compression test specimen and (c) short and thick beam shear test setup. 

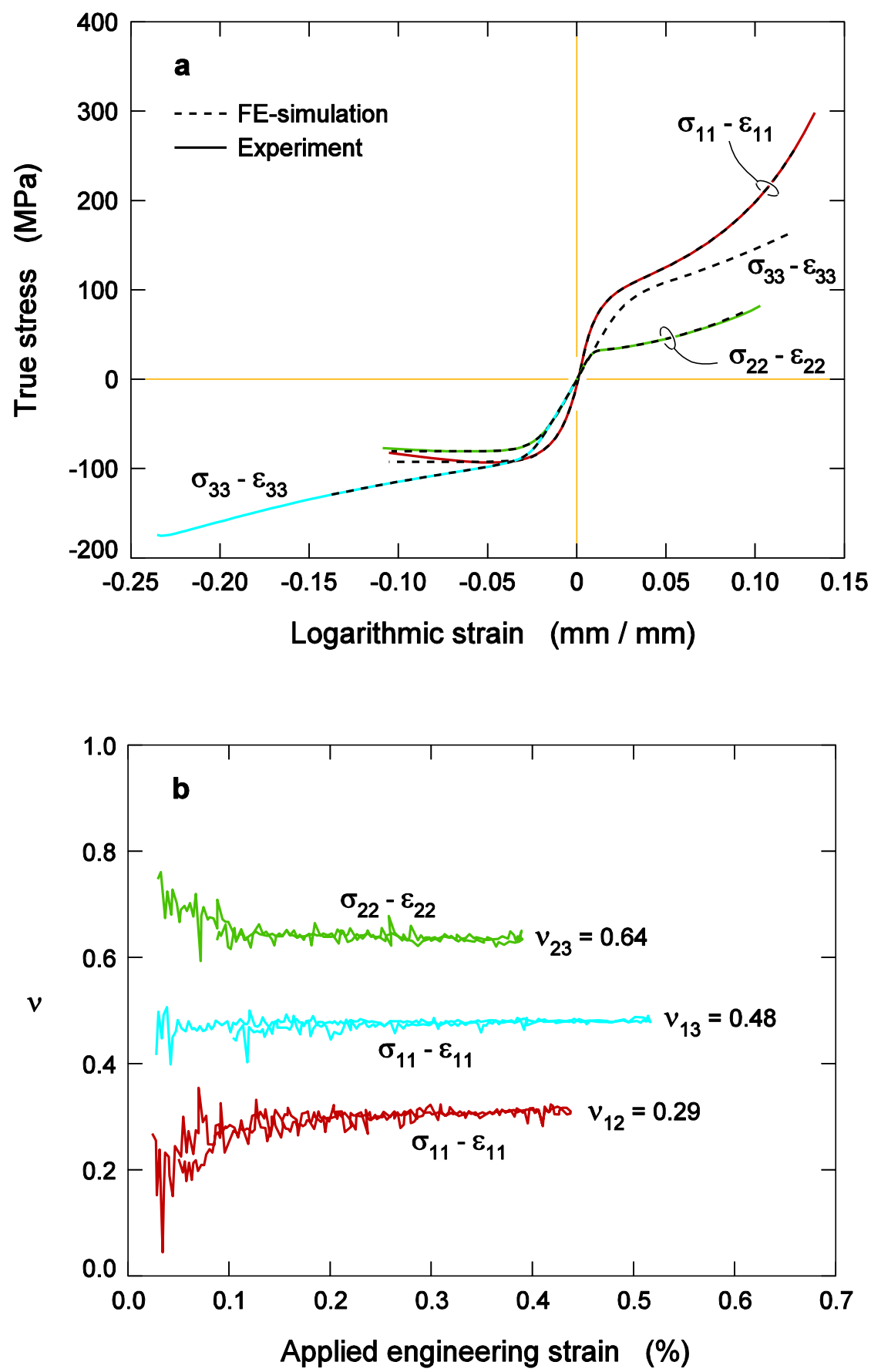

Figure 3: (a) The measured true stress versus strain responses of the PET composites for loading in the three principal material directions $x_{1}, x_{2}$ and $x_{3}$ at an applied strain rate $10^{-4} \mathrm{~s}^{-1}$. The FE predictions using the proposed constitutive model are included. (b) The measured Poisson's ratios as a function of applied strain within the elastic regime of loading. The tests from which these ratios are inferred are indicated in each case. 

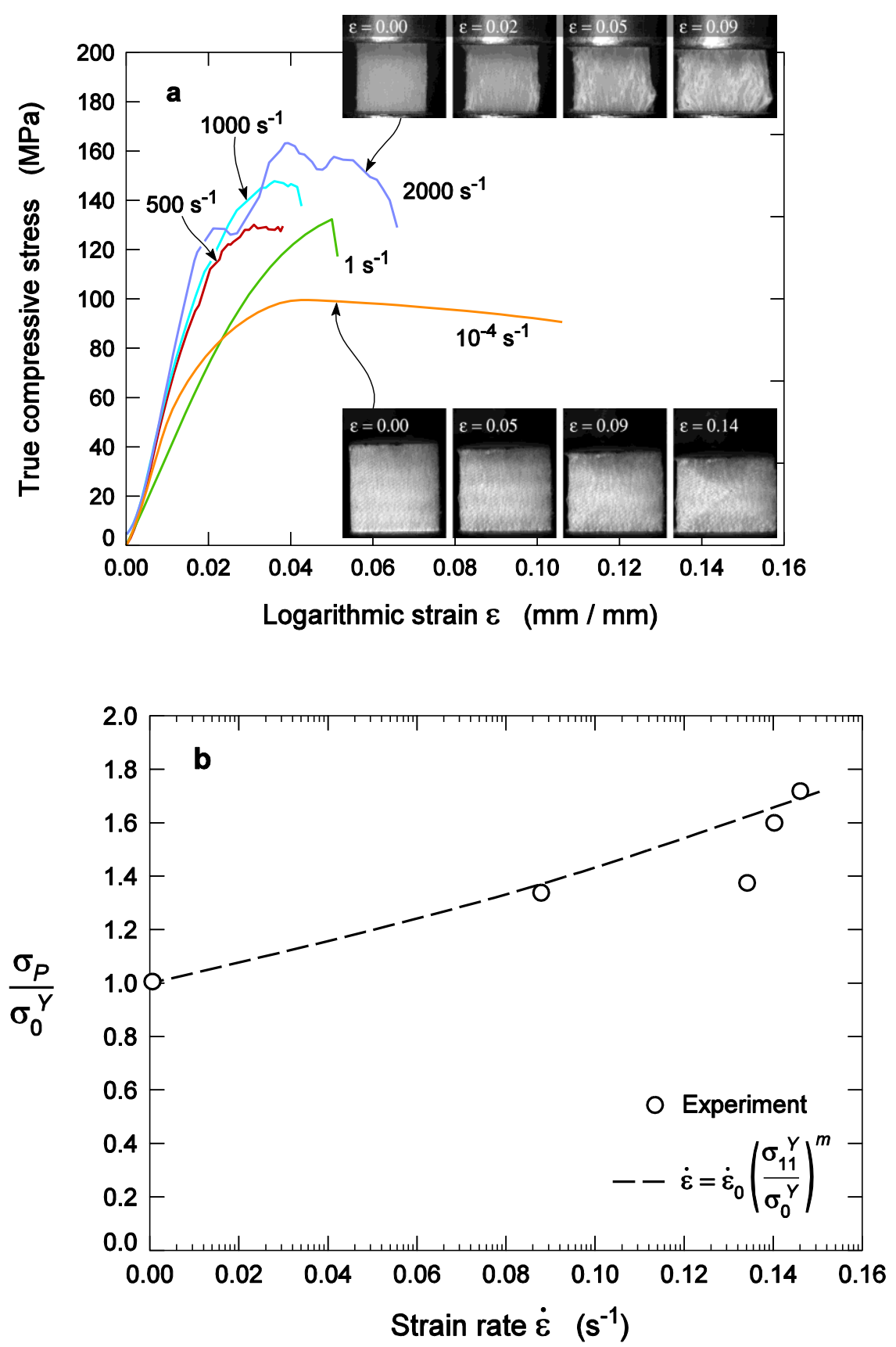

Figure 4: (a) The measured compressive stress versus strain response of the PET composites for loading in the $x_{1}$ direction over an applied strain rate range $10^{-4} \mathrm{~s}^{-1} \leq$ $\dot{\varepsilon} \leq 2000 \mathrm{~s}^{-1}$. Montages of photographs showing the deformation of the specimens for loading at $\dot{\varepsilon}=10^{-4} \mathrm{~s}^{-1}$ and $2000 \mathrm{~s}^{-1}$ are included. (b) The measured peak compressive strength $\sigma_{p}$ in the $x_{1}$ direction normalized by the quasi-static strength $\sigma_{0}^{Y}=$ $104 \mathrm{MPa}$ as a function of the applied strain rate $\dot{\varepsilon}$. 


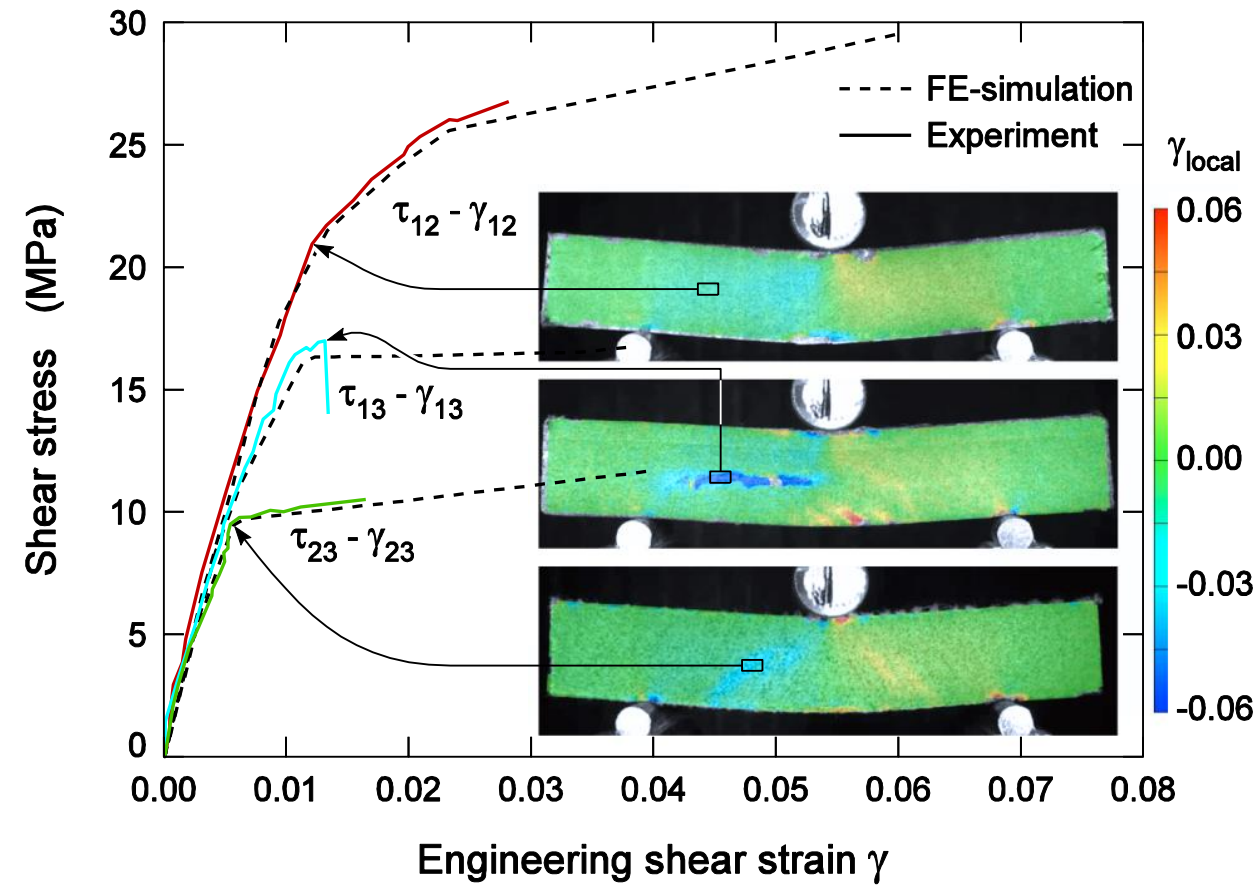

Figure 5: The measured applied shear stress versus engineering shear strain responses of the PET composites at an applied strain rate of $10^{-4} \mathrm{~s}^{-1}$. The FE predictions using the proposed constitutive model are included and the inset shows contours of the measured shear strains $\gamma_{i j}$ (via DIC) on the surfaces of the specimens at the instants indicated. 

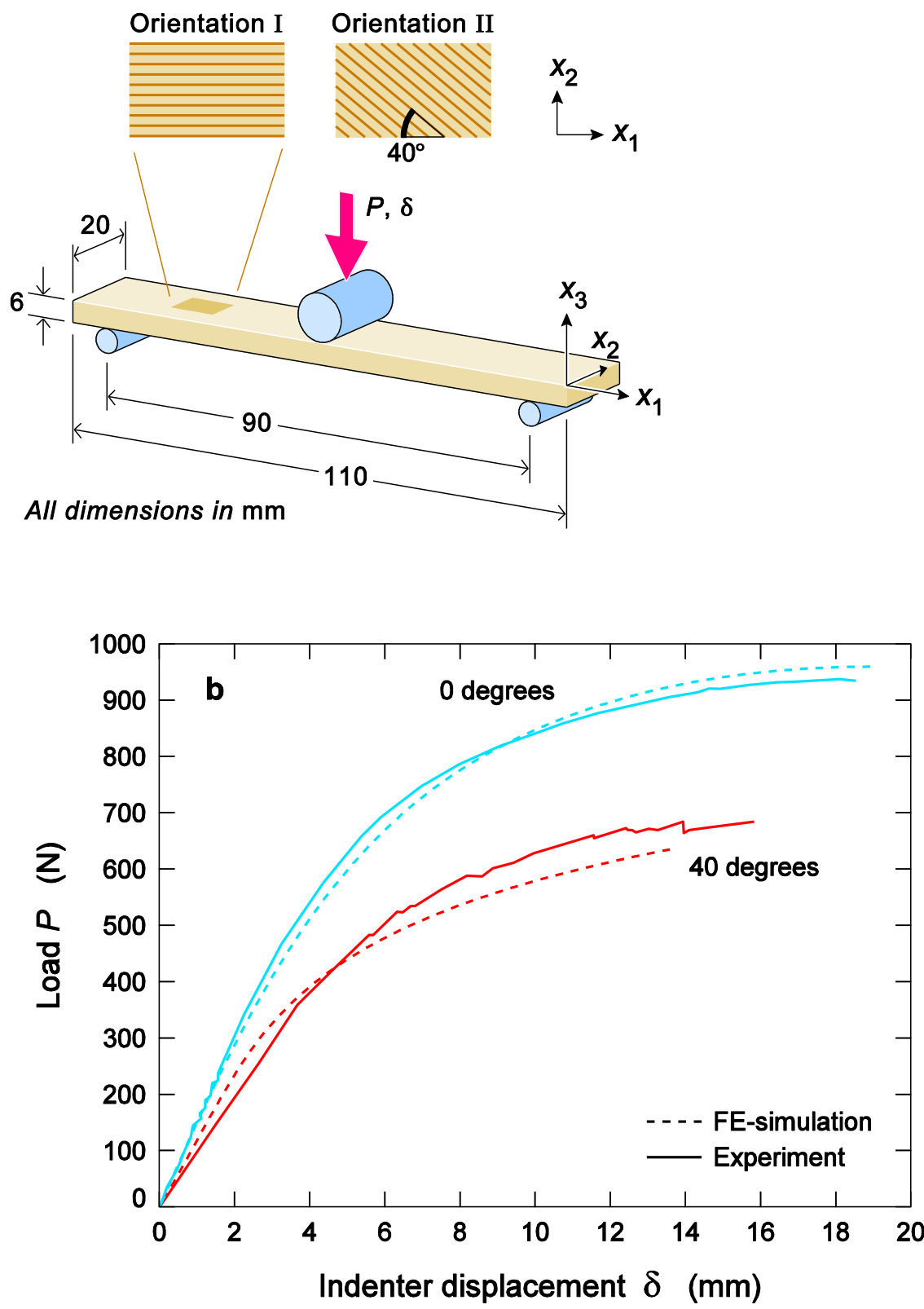

Figure 6: (a) Sketch of the three-point bend loading of the PET composite beams. All dimensions are in $\mathrm{mm}$ and the inset shows the two orientation of the material with respect to the beam axes. (b) The measured applied load $P$ versus displacement $\delta$ curves along with the corresponding FE predictions. 
a

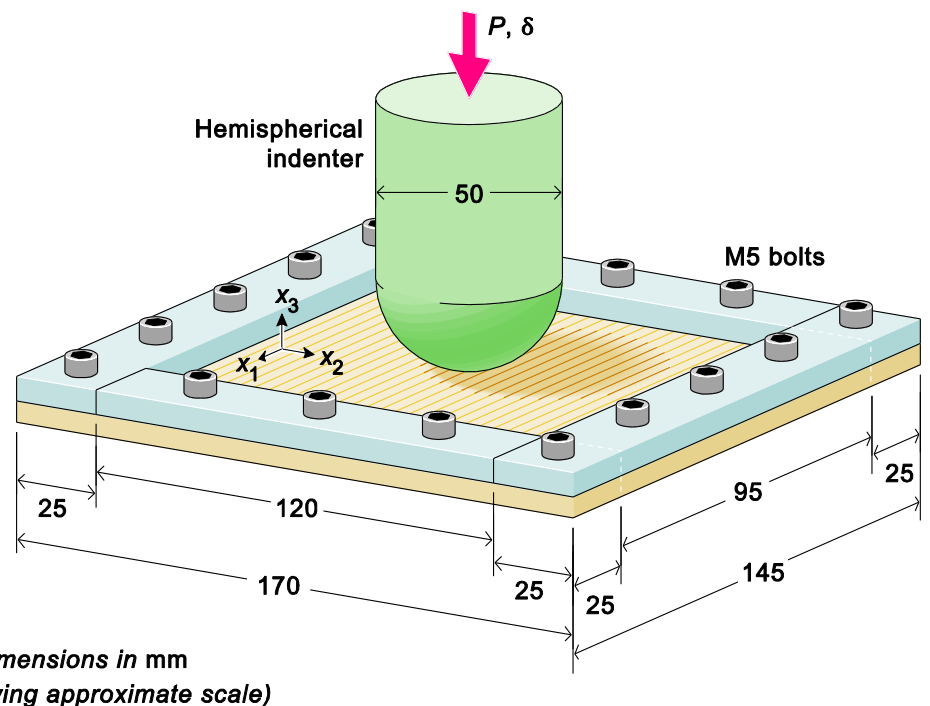

(drawing approximate scale)

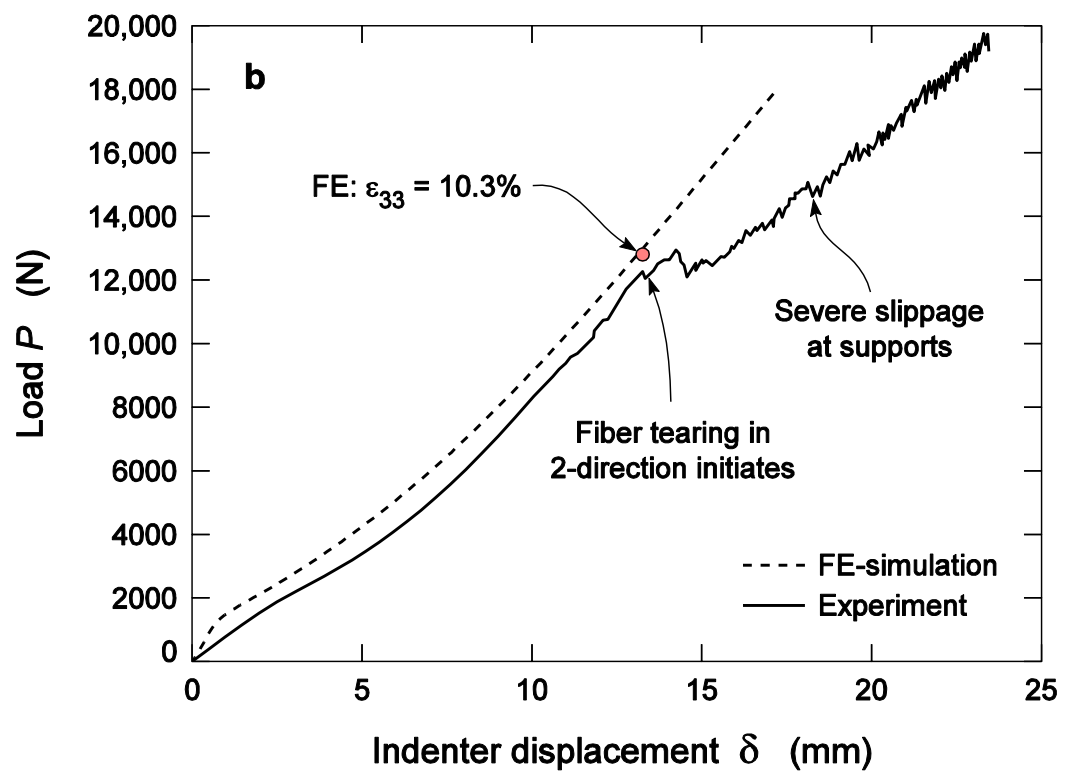

Figure 7: (a) Sketch of the central loading of PET composite plate. All dimensions are in $\mathrm{mm}$ and the orientation of the $x_{1}$ material direction is indicated. (b) The measured applied load $P$ versus displacement $\delta$ curves along with the corresponding FE predictions. 


\begin{tabular}{ccccccccc}
\hline$E_{11}$ & $E_{22}$ & $E_{33}$ & $G_{12}$ & $G_{23}$ & $G_{13}$ & $v_{12}$ & $v_{23}$ & $v_{13}$ \\
$(\mathrm{MPa})$ & $(\mathrm{MPa})$ & $(\mathrm{MPa})$ & $(\mathrm{MPa})$ & $(\mathrm{MPa})$ & $(\mathrm{MPa})$ & & & \\
\hline 6000 & 4000 & 3300 & 2000 & 2000 & 2000 & 0.29 & 0.64 & 0.48
\end{tabular}

Table 1: Measured elastic properties for PET composite. 